\title{
Relaciones dinámicas del producto y el empleo en México: una evaluación de sus componentes permanentes y transitorios
}

\author{
Alejandro Islas C. y Willy W. Cortez
}

RESUMEN

Las conclusiones de estudios anteriores sobre la relación entre los componentes cíclicos del producto y el desempleo en México permiten suponer que esta es muy similar a la existente en la economía de los Estados Unidos de América. Ello indicaría que las relaciones dinámicas entre el producto y el mercado de trabajo de las dos economías tienen muchos rasgos en común, lo que es sorprendente porque, de hecho, no concuerda con la caracterización del mercado laboral de México. La aplicación de la metodología propuesta originalmente por Clark (1989) permitió concluir que la correlación entre los componentes transitorios del producto y el desempleo es mucho menor de lo que se creía.

PALABRAS CLAVE

CLASIFICACIÓN JEL

AUTORES
Mercado de trabajo, desempleo, producción, modelos matemáticos, México

$\mathrm{C} 32, \mathrm{E} 23, \mathrm{E} 24, \mathrm{E} 32$

Alejandro Islas C. es profesor numerario III C en el Departamento de Estadística del Instituto Tecnológico Autónomo de México. aislas@itam.mx

Willy W. Cortez es profesor-investigador titular C en el Departamento de Métodos Cuantitativos del Centro Universitario de Ciencias Económico-Administrativas (cucea) de la Universidad de Guadalajara, Jalisco, México.wcortez@cucea.udg.mx 


\section{I}

\section{Introducción}

Entre los años 1987 y 2008, México registró tasas muy fluctuantes de desocupación abierta, que oscilaron entre un $5,2 \%$ en el primer trimestre de 1987 , un $2,6 \%$ en el segundo trimestre de 1991 y un $8,2 \%$ en el tercer trimestre de 1995, en el que alcanzó su nivel más alto. Posteriormente, esta tasa se redujo a un 2,3\% en el cuarto trimestre de 2000 y desde entonces ha mostrado una tendencia ascendente. Estas variaciones del valor medio del desempleo se vieron acompañadas por cambios en la volatilidad del empleo, que reflejan variaciones de la dinámica del mercado laboral. La desviación estándar del desempleo se mantuvo en torno de un 0,176 entre el primer trimestre de 1987 y el cuarto trimestre de 1994, mientras que entre el primer trimestre de 1995 y el cuarto trimestre del año 2000 aumentó a cerca de un 0,394 , para luego disminuir a un 0,148 en el período comprendido entre el primer trimestre de 2001 y el primero de 2007.

Estas fluctuaciones del desempleo han coincidido con variaciones del producto en dirección contraria; es decir, cuando el desempleo fue inferior a su tendencia a largo plazo, el producto fue superior a esta y viceversa. Chavarín (2001) y Loria y Ramos (2007), entre otros, han determinado que cuando se produce una variación del desempleo de un punto porcentual, el producto muestra un decrecimiento negativo de un $2,3 \%$ a un $2,7 \%$. La regularidad empírica entre las variaciones del desempleo y el producto, conocida como "ley de Okun", es un componente fundamental de la teoría macroeconómica keynesiana debido al vínculo que establece entre el producto y el mercado de trabajo ${ }^{1}$.

En muchos artículos se destaca la validez de la ley de Okun como herramienta para la adopción de políticas. Por ejemplo, Knotek (2007) considera que se la puede aplicar como una simple regla general para determinar la medida en que una cierta tasa de desempleo se traduce en un cierto ("x") incremento del producto. La aplicación de esta ley también ayuda a pronosticar la tasa de desempleo. Por otra parte, Balakrishnan, Das y Kannan (2010) recurrieron a la ley de Okun como

$\square$ Los autores agradecen los valiosos comentarios de los integrantes de la sede subregional de la CEPAL en México y a los participantes en el Seminario Aleatorio del Instituto Tecnológico Autónomo de México.

1 Prachowny (1993) indica que la curva de la oferta agregada se calcula a partir de la curva de Phillips, complementada por la ley de Okun. marco explicativo de la dinámica del desempleo en un grupo de países adelantados durante la última recesión.

En la literatura sobre el tema se observa un particular interés de los economistas por determinar la regularidad de las estimaciones basadas en la ley de Okun. Un rápido examen de varios estudios sobre los Estados Unidos de América y otros países desarrollados indica que el coeficiente presenta significativas diferencias entre un país y otro y en distintos períodos. También se ha demostrado que el coeficiente de Okun varía de acuerdo con el horizonte temporal empleado para medir la relación entre el desempleo y el producto (contemporáneo, o a corto o largo plazo) (Weber, 1995).

La variación del coeficiente de Okun de un país a otro puede atribuirse a varios factores. Blanchard y Quah (1989), entre otros, sostienen que este coeficiente es híbrido, porque depende de las perturbaciones que afecten a una economía, es decir, de las restricciones de la oferta y la demanda ${ }^{2}$. En los estudios internacionales sobre la materia se demuestra categóricamente la inestabilidad del coeficiente. Schnabel (2002) observó que en el período transcurrido entre 1990 y 2000 este registró importantes cambios en una muestra de países industrializados, pero que estos cambios no fueron homogéneos; de hecho, en algunos de esos países el incremento del producto mostró una mayor sensibilidad a las fluctuaciones del desempleo. Cazes, Verick y Al Hussami (2011) y Balakrishnan, Das y Kannan (2010) ofrecen pruebas de los heterogéneos efectos de la crisis económica de 2008 en los mercados de trabajo de los países miembros de la Organización de Cooperación y Desarrollo Económicos (OCDE); específicamente, algunos de ellos, como España y los Estados Unidos de América, se han visto afectados por marcadas variaciones de la tasa de desempleo, mientras que en otros estas fueron menores, como ocurrió en Alemania, Italia y el Japón. En estudios recientes se ofrecen nuevas pruebas de que el coeficiente de Okun no es simétrico, sino que muestra diferentes valores en los períodos de auge y recesión (Jardin y Gaétan, 2011).

\footnotetext{
2 Los autores llegaron a la conclusión de que el coeficiente de Okun es mucho menor cuando la perturbación proviene de la demanda que cuando se origina en la oferta.
} 
Lo que se conoce de la relación dinámica entre producto y desempleo en la economía de México es más bien limitado. En la literatura se sostiene que el coeficiente de ambos es similar a lo indicado por los cálculos originales de Okun para la economía de los Estados Unidos de América (Chavarín, 2001; Loria y Ramos, 2007). Estas conclusiones son sorprendentes, porque supondrían que el mercado laboral mexicano es tan flexible como el de los Estados Unidos de América, cuando en realidad - como lo demuestran distintas medidas de la flexibilidad - es uno de los más rígidos entre los países de la oCDE y de América Latina ${ }^{3}$.

En un estudio de 13 países de la región, González Anaya (2002) llegó a la conclusión de que en México el coeficiente de Okun era uno de los más bajos de dichos países. Según sus estimaciones, este se acercaba más a los observados en Europa y el Japón, lo que el autor atribuyó en parte a la mayor flexibilidad de los salarios reales existente en México.

Los estudios de Chavarín (2001) y Loria y Ramos (2007) tienen dos limitaciones adicionales. En primer lugar, desde el punto de vista estadístico, en ambos casos se estimó el coeficiente de Okun mediante un método que consta de dos pasos, lo que podría dar origen a

3 Según la oCDE, el índice de protección del empleo de México fue de un 3,2 en los años noventa y la década de 2000, lo que se compara con un 0,21 en la economía de los Estados Unidos de América. resultados sesgados e ineficientes (Sinclair, 2009). En segundo término, en ninguno de estos estudios se ofrece una visión de la dinámica entre la producción y el empleo o del funcionamiento del mercado de trabajo que pudiera explicar la magnitud del coeficiente de Okun en el caso de México.

Como se verá más adelante, la estimación del coeficiente de Okun plantea varios problemas. El alcance del presente estudio es bastante específico, por el hecho de que el interés en él es ofrecer una estimación puntual, no sesgada y eficiente del coeficiente. Para hacerlo, el trabajo se aparta del procedimiento habitual en dos etapas para adoptar la metodología propuesta originalmente por Clark (1989). Además, se establece una relación entre la magnitud del coeficiente estimado y las condiciones imperantes en el mercado de trabajo de México, y se explica adecuadamente por qué se puede considerar que las estimaciones son razonables o aceptables.

El presente estudio se divide en seis secciones incluida esta Introducción. En la sección II se presenta una reseña de algunos estudios de estimación del coeficiente de Okun, mientras que en la sección III se analizan brevemente el carácter del mercado laboral mexicano y la información que aporta la técnica de estimación de Clark. En la sección IV se describe el modelo econométrico utilizado para estimar la relación entre producto y desempleo y en la sección $\mathrm{V}$ se examinan los principales resultados del estudio. Por último, en la sección VI se presentan algunas conclusiones finales.

\section{II}

\section{Ley de Okun}

Lo que se proponía Okun en el estudio publicado en 1962 era estimar el costo del desempleo en términos del producto potencial. Desde entonces, el objetivo de su investigación se ha convertido en un área fértil para el análisis de la dinámica del producto y el desempleo, y de la relación entre ambos a lo largo de los ciclos económicos.

En su influyente estudio, Okun (1962) estimó que el aumento del desempleo en un punto porcentual se traduciría en una disminución del incremento del producto cercana al 3,3\%. Aunque pocos investigadores lo han advertido, la medición de los efectos del desempleo en el producto potencial se basa en el supuesto de que la tasa de desempleo resume el comportamiento de otras variables (promedio de horas trabajadas, tasas de participación y productividad de la mano de obra, entre otras) o está correlacionada con estas. También se podría decir que el desempleo puede considerarse una variable que mide de forma aproximada todas las formas en que el producto se ve afectado por los recursos inactivos. Esta suposición es muy importante para calcular y predecir que el coeficiente fijo entre la variación del desempleo y el incremento del producto sea fijo, porque en caso de no ser cierta no cabría esperar que esto fuera así. Además, el cambio tecnológico, los cambios en las instituciones que conforman el mercado de trabajo, la variación de las tasas de participación y los cambios demográficos, entre otros, modificarían el coeficiente.

Los estudios publicados a la fecha permiten concluir que la relación entre los componentes cíclicos del producto 
y el desempleo es bastante compleja e inestable, porque depende de una serie de variables y, por lo tanto, no se debería esperar que sea idéntica entre los distintos países ${ }^{4}$. Para explicar esta situación, en este estudio se define el desempleo como la diferencia entre la oferta y la demanda de mano de obra a una tasa salarial dada, por lo que sus variaciones obedecen a fluctuaciones de la oferta o la demanda de mano de obra o de ambas. La oferta de mano de obra depende de las variables demográficas y de las instituciones que integran el mercado de trabajo 5 . Por otra parte, la demanda de mano de obra depende del progreso técnico y de las condiciones imperantes en el mercado de bienes. En el corto plazo, el empleo real también depende de la capacidad de las empresas para modificar el horario de trabajo y la productividad de la fuerza laboral. Por lo tanto, no se sabe con precisión si un cambio en la demanda de productos daría lugar a un cambio inmediato del empleo y, por consiguiente, del desempleo. Por ejemplo, si la demanda agregada registra un aumento y se responde a este con un incremento del horario de trabajo o un alza de la productividad de la mano de obra o con ambos, el empleo (desempleo) no debería aumentar (disminuir) obligatoriamente. No se puede saber a priori a qué ritmo se ajustarían estas variables entre los distintos países y a lo largo del tiempo. Lo que sí se sabe es que el ajuste no sería uniforme en todos los casos.

La ley de Okun se define comúnmente en términos del efecto que ejerce en el incremento del producto una variación del desempleo de un punto porcentual. Según la teoría keynesiana, el desempleo depende de las condiciones existentes en el mercado de bienes, lo que también podría expresarse diciendo que el desempleo es la variable dependiente y el producto es la variable independiente ${ }^{6}$. En el pasado, los economistas calcularon el desempleo en función del producto para luego suponer que el coeficiente de Okun era el inverso del parámetro estimado. Ahora se sabe que este cálculo es incorrecto, porque - como se ha explicado- la relación entre las dos variables no es lineal y, además, es probable que midan distintos fenómenos. Barreto y Howland (1993),

\footnotetext{
${ }^{4}$ En un estudio de los países de la oCDE, Lee (2000) determinó que las estimaciones de Okun también son sensibles al modelo que se elija (lo que incluye la primera diferencia y las especificaciones de brechas). 5 Las variables demográficas son, entre otras, el crecimiento de la población y la participación de las mujeres en el mercado de trabajo. Entre las instituciones del mercado laboral cabe mencionar los factores que influyen en las preferencias de los trabajadores respecto de la combinación de trabajo y descanso, las leyes de protección del empleo, los hábitos laborales y la dedicación al trabajo.

6 A juicio de Keynes, la demanda de mano de obra es una demanda derivada.
}

que se cuentan entre los primeros en advertir este hecho, consideran aconsejable otorgar especial importancia al sentido de la regresión. A su juicio, Okun había supuesto erróneamente que se podía determinar la relación en ambos sentidos. Específicamente $\Delta \mathrm{u}=f(\Delta y)$ puede relacionarse con restricciones de la demanda o de la oferta, o de ambas, mientras que $\Delta y=g(\Delta \mathrm{u})$ puede vincularse a perturbaciones demográficas y a restricción de la oferta. Desde el punto de vista teórico, no hay ningún motivo para suponer que existe una relación aritmética o algebraica entre los dos coeficientes, independientemente del efecto de retroalimentación que pueda producirse entre ellos.

En los estudios sobre la materia se identifican tres técnicas de estimación: i) estimación del coeficiente mediante el procedimiento convencional en dos etapas; ii) estimación del coeficiente de Okun conforme a un modelo bivariado, que permite el cálculo conjunto del componente cíclico y el componente tendencial, y iii) estimación del coeficiente de Okun basada en el supuesto de que este varía a lo largo del tiempo.

La estimación convencional del coeficiente de Okun se realiza en dos etapas. En la primera se elimina el componente permanente de las series y en la segunda se estima la correlación entre los componentes transitorios del producto y el desempleo. Para calcular el componente permanente se emplean distintas técnicas, que incluyen el uso de mínimos cuadrados ordinarios (MCO) o del filtro de Hodrick-Prescott. En algunos casos, simplemente se ha eliminado el componente permanente no observable mediante el uso de las primeras diferencias de las series $^{7}$. Una vez estimado el componente permanente no observable, se calcula el componente transitorio mediante una operación consistente en restar el primero de la serie observada. En la segunda etapa del proceso se recurre a los MCo para estimar el coeficiente de Okun.

Sinclair (2009) sostiene que el coeficiente calculado con esta metodología es sesgado e ineficiente por dos motivos. En primer lugar, dada la existencia de una correlación entre los componentes permanentes y transitorios de las dos series, sería más eficiente estimar conjuntamente sus componentes cíclicos. En segundo término, dada la correlación entre el error de medición de la variable independiente y la variable dependiente, las estimaciones basadas en los MCO están sesgadas y son inconsistentes. Por lo tanto, sería más adecuado utilizar la correlación estimada que la correlación entre las estimaciones.

\footnotetext{
7 Cuando una de las series es estacionaria, es decir $\mathrm{I}(0)$, la primera etapa puede ser innecesaria.
} 
Los modelos bivariados — que se emplean para calcular conjuntamente los componentes permanentes y transitorios del desempleo y el producto- surgieron como una reacción a la metodología de Nelson y Plosser (1982) de exclusión del componente no estacionario mediante la diferenciación de la serie, lo que convierte a la tendencia en una caminata aleatoria, en lugar de una línea recta. Clark (1987) señala que este enfoque tiene dos limitaciones. La primera de ellas es que las pruebas de no estacionariedad arrojan resultados con bajo poder en comparación con alternativas plausibles. La segunda se debe a que el análisis se basa en el fuerte supuesto de que la función de autocorrelación de la primera diferencia del producto es igual a cero después del primer rezago.

Clark (1987) propuso un nuevo análisis del producto de los Estados Unidos de América, en el que se distinguen los dos componentes no observables de las series: la tendencia no estacionaria y el componente cíclico estacionario. Este análisis tiene como marco de referencia el modelo estado-espacio, que permite una especificación más general del componente tendencial. Clark (1989) usó el filtro de Kalman y el método de máxima verosimilitud para calcular el componente no estacionario permanente y el componente estacionario cíclico del incremento del producto y el desempleo en seis países desarrollados ${ }^{8}$. El autor encontró pruebas convincentes de que el componente estacionario estimado del producto está estrechamente vinculado al componente cíclico del desempleo. Por su parte, Evans (1989) recurrió a un modelo bivariado de vectores autorregresivos (VAR) para describir la relación entre producto y desempleo, estimar el grado de persistencia de las innovaciones del producto y descomponer el producto en tendencia y ciclo. Este autor llega a la conclusión de que un análisis bivariado revela la existencia de una retroalimentación entre el incremento del desempleo y el producto, así como una relación contemporánea negativa entre crecimiento del producto e innovaciones en el desempleo.

El examen de la relación entre los componentes transitorios y los componentes permanentes del producto interno bruto (PIB) real es relevante, porque permite determinar a la variación de cuáles de ellos obedece la variación observada del PIB. Asimismo, puede facilitar la estimación de las relaciones entre los dos tipos de componentes.

La tercera técnica de estimación se vincula a la variación temporal del coeficiente. A partir de mediados de

8 Alemania, Canadá, Estados Unidos de América, Francia, Japón y Reino Unido de Gran Bretaña e Irlanda del Norte. los años noventa son cada vez más comunes los estudios destinados a determinar si el coeficiente de Okun es o no estable. Por ejemplo, Prachowny (1993) considera que la relación de 3 a 1 entre el producto y el desempleo solo se mantiene debido a que tienden a aumentar factores tales como el número de horas laborables por semana, la oferta inducida de mano de obra y la productividad. Una conclusión importante del artículo de Prachowny es que si cualquiera de estos otros factores cambia y todos los demás elementos se mantienen constantes, la relación entre el producto y el desempleo también debería cambiar.

Autores como Knotek (2007) y Balakrishnan, Das y Kannan (2010) ofrecen pruebas de que, a diferencia de lo que indican las conclusiones de otros estudios, la relación entre el producto y el desempleo no ha sido constante. En un estudio sobre la economía de los Estados Unidos de América que abarca el período comprendido entre 1960 y 2007, Knotek observó una fluctuación del coeficiente de Okun de -0,067 en 1975 a alrededor de $-0,088$ en 1995 y a $-0,04$ en 2007 , lo que revela que este aumentó considerablemente en la primera década del presente siglo.

La investigación sobre la dinámica del desempleo en varios países industrializados realizada por Balakrishnan, Das y Kannan (2010) les permitió concluir que el coeficiente de Okun mostró grandes cambios entre los Estados miembros de la oCDE en el período estudiado (1980-2008). En particular, observaron que, en los casos del Reino Unido de Gran Bretaña e Irlanda del Norte y Suecia, el coeficiente registró un aumento sostenido en términos absolutos, mientras que en Alemania y los Estados Unidos de América presentó fluctuaciones que no responden a ningún patrón definido. Los autores sostienen que los cambios institucionales en los mercados de trabajo y los cambios tecnológicos y demográficos son los causantes de las variaciones del coeficiente en los países desarrollados que se incluyeron en el estudio. En un estudio reciente de Cazes, Verick y Al Hussami (2011) se presentan pruebas de que la repercusión de la crisis financiera de 2008 en el desempleo no fue igual en los Estados Unidos de América que en los países europeos. Las diferencias entre ellos se atribuyen, entonces, a la disímil evolución del coeficiente de Okun.

Los autores también llegaron a la conclusión de que el efecto de las fluctuaciones del producto en el desempleo es asimétrico, es decir, que el comportamiento del coeficiente no es igual en los episodios de recesión que en los de recuperación. Crespo (2003) observó que en la economía de los Estados Unidos de América el 
efecto contemporáneo del crecimiento en el desempleo es mucho mayor en los períodos de recesión que en los de expansión. También observó que las perturbaciones que inciden en el desempleo suelen ser más persistentes en etapas expansivas. En un estudio de 16 países europeos, Jardin y Gaétan (2011) encontraron pruebas de que el desempleo es más sensible al crecimiento del producto en períodos de contracción de la economía que en episodios de expansión.

En la siguiente sección se analizan algunas de las principales características del mercado de trabajo de México y se presentan dos visiones contrastantes acerca de su naturaleza, lo que es relevante porque facilita la comprensión de las conclusiones empíricas.

\section{III}

\section{¿Cuán flexible es el mercado laboral de México?}

El sentido común hace suponer que la magnitud del coeficiente de Okun de un determinado país depende de la dinámica del mercado de trabajo, impuesta por el marco institucional y el proceso de cambio tecnológico. Los estudios sobre las características del mercado laboral de México se pueden clasificar en dos categorías en virtud de su conceptualización. Algunos autores consideran que este mercado está muy regulado por leyes que dificultan la creación de empleo. En este caso, el incremento del producto no se traduciría obligatoriamente en marcadas variaciones del desempleo, sino en fluctuaciones de los salarios reales (Heckman y Pagés, 2000; Gill, Montenegro y Dömeland, 2001). En los períodos de recesión y debido a la rigidez de las leyes laborales federales y de los sindicatos, a las empresas les resultaría extremadamente difícil despedir trabajadores ${ }^{9}$. También se considera que las normas sobre estabilidad laboral, en las que se prevén indemnizaciones por despido, elevan el costo que este tiene para las empresas. Dicho costo representa un desincentivo a la supresión de trabajadores en casos de perturbaciones negativas y limita la creación de empleo en períodos de expansión. Heckman y Pagés (2000) observaron que México tiene uno de los índices más altos de estabilidad laboral de América Latina, lo que significa que su mercado de trabajo es uno de los más regulados de la región ${ }^{10}$. En caso de que esta rigidez del mercado se manifieste tanto en los períodos de expansión como

\footnotetext{
9 En diciembre de 2012, el Congreso de México aprobó una nueva legislación laboral según la cual se otorga a las empresas mucho más flexibilidad para la contratación y el despido de trabajadores.

10 Asimismo, la oCDE determinó que de todos sus países miembros, México es el que presenta el índice más alto de protección del empleo, referido tanto a los contratos permanentes como a los contratos temporales.
}

de recesión, cabría esperar que la correlación entre los componentes transitorios del producto y el desempleo sea baja, es decir, que el coeficiente de Okun sea bajo.

De acuerdo con la visión opuesta del mercado laboral, desde mediados de los años ochenta, cuando México comenzó a aplicar una nueva estrategia de desarrollo basada en la liberalización económica y comercial, un número cada vez mayor de empresas han adoptado nuevos mecanismos que les permiten adaptarse mejor a las fluctuaciones económicas (De la Garza, 2005). Entre otras cosas, estas recurren con mucho mayor frecuencia a los contratos cortos y las contrataciones externas, a fin de reducir los costos derivados de la estabilidad laboral. El cambio es especialmente marcado en la industria maquiladora y el sector de servicios, los dos sectores más dinámicos de la economía de México desde fines de los años ochenta (Marshall, 2004) ${ }^{11}$. Por consiguiente y conforme con este criterio, se podría pensar que el coeficiente de Okun es suficientemente alto como para que las variaciones del crecimiento del producto den origen a amplias variaciones de las tasas de desempleo.

En este debate está implícito el reconocimiento de la existencia de un amplio sector informal que absorbe a cerca de la mitad de los trabajadores empleados de México (Loayza y Sugawara, 2009). Dado que la informalidad es una variable no observable, el tamaño del mercado informal de trabajo se ha estimado indirectamente ${ }^{12}$.

Calderón (2000) observó una estrecha relación entre los mercados de trabajo formal e informal de México, idea que fue corroborada en estudios más recientes de

\footnotetext{
${ }^{11}$ La industria maquiladora está integrada por plantas de ensamblaje cuya producción se destina fundamentalmente a la exportación.

12 Loayza y Sugawara (2009) presentan una breve descripción de algunos de los métodos empleados.
} 
Alcaraz, Chiquiar y Ramos-Francia (2008) y Alcaraz (2009). Estos autores encontraron pruebas de que la tasa de transición entre el empleo formal e informal es superior a la existente entre los sectores manufacturero y de servicios. A su juicio, esta mayor movilidad obedecería a la existencia de rigidez institucional en el mercado laboral de México.

En la sección II se explicó la razón por la que en determinadas circunstancias las variaciones del producto no se traducen en variaciones del desempleo y viceversa. La estrecha relación que existe entre el extenso mercado informal de trabajo y el mercado formal representa un canal adicional mediante el cual las fluctuaciones del producto no conducen necesariamente a variaciones del desempleo abierto y viceversa. La misma idea se podría expresar diciendo que la existencia de un amplio mercado informal de trabajo modificaría la relación esperada entre los componentes cíclicos del producto y el desempleo. Lo que se observa, en cambio, es que una variación del producto acentúa la movilidad entre el sector formal y el informal, en tanto que la tasa de desempleo se mantiene invariable. Si se descompone la tasa de empleo en tasa de empleo formal $\left(e_{f}\right)$ y tasa de empleo informal $\left(e_{i n f}\right)$, entonces, la siguiente proposición es verdadera:

$$
\begin{gathered}
\mathrm{u}=1-e_{f}-e_{i n f} \\
\Delta \mathrm{u}=-\Delta e_{f}-\Delta e_{i n f}
\end{gathered}
$$

\footnotetext{
13 También podría ocurrir que la importancia relativa del sector informal fuera relativamente baja.
}

Ante la considerable regulación del empleo formal, la mayor parte de las variaciones del desempleo sería absorbida por fluctuaciones del empleo informal. Además, como en las estadísticas oficiales de México sobre el mercado de trabajo se considera que el empleo informal es una forma de empleo, la correlación entre las variaciones del producto y el desempleo sería más bien baja, a menos que el sector informal no sea suficientemente flexible ${ }^{13}$. Esta conclusión es válida incluso dada la rigidez institucional mencionada por Heckman y Pagés (2000). El mismo Okun indica que el valor del coeficiente depende de una serie de supuestos bien fundamentados sobre la productividad laboral, el promedio de horas trabajadas y las tasas de participación ${ }^{14}$.

Una vez analizada la relación entre los componentes transitorios del desempleo y el producto, habría que determinar qué forma adopta la relación entre sus componentes permanentes. En este estudio, la suposición es que la relación de equilibrio a largo plazo está medida por la correlación entre los componentes permanentes de ambas series. Si esto es cierto, el coeficiente de Okun sería menor debido a que en el largo plazo hay variables que no se mantienen fijas, entre otras, la tasa de utilización del capital, la tecnología, las tasas de participación y la productividad del trabajo.

${ }^{14}$ Por lo tanto, si cualquiera de estas variables cambiara, el coeficiente no se mantendría fijo sino que iría cambiando paulatinamente.

\section{IV}

\section{El modelo del producto y la tasa de desempleo}

En esta sección se presenta el modelo de componentes permanentes y transitorios del producto y la tasa de desempleo desarrollado por Clark (1989) y Sinclair (2009):

$$
\begin{gathered}
y_{t}=\tau_{y_{t}}+c_{y_{t^{\prime}}} \\
\tau_{y_{t}}=\mu_{y}+\tau_{y_{t-1}}+\eta_{y_{t^{\prime}}} \\
u_{t}=\tau_{u_{t}}+c_{u_{t^{\prime}}} \\
\tau_{u_{t}}=\mu_{u}+\tau_{u_{t-1}}+\eta_{u_{t}}
\end{gathered}
$$

En este modelo, el producto $\left(y_{t}\right)$ y la tasa de desempleo $\left(u_{t}\right)$ son la suma de dos componentes. El primero de ellos $\left(\tau_{i_{t}}, i=y, u\right)$ es el componente permanente, que corresponde a la constante calculada una vez excluidos todos los cambios transitorios. El segundo $\left(c_{i}, i=y, u\right)$ es el componente transitorio, que refleja todos los cambios de ese tipo y que supuestamente es estacionario. Asimismo, se supone que las componentes de tendencia son caminatas aleatorias a fin de incluir los movimientos permanentes de la serie. Por otra parte, los componentes transitorios $\left\{\left(c_{y_{t}}, c_{u_{t}}\right)\right\}$ constituyen un proceso estocástico bivariado y estacionario. 
Para completar la caracterización del producto y las tasas de desempleo, se supuso que las desviaciones transitorias de los valores de equilibrio siguen un proceso estacionario $(\operatorname{VAR}(\mathrm{p}))$ :

$$
\begin{gathered}
\Phi(L) \underline{c_{t}}=\underline{\varepsilon_{t}} \\
\text { donde } c_{t}=\left(\begin{array}{l}
c_{y_{t}} \\
c_{u_{t}}
\end{array}\right), \\
\left.\underline{\varepsilon_{t}}=\left(\begin{array}{l}
\varepsilon_{y_{t}} \\
\varepsilon_{u_{t}}
\end{array}\right) \text { i.i.d }\left(\begin{array}{l}
0 \\
0
\end{array}\right),\left(\begin{array}{cc}
\sigma_{\varepsilon_{y}}^{2} & \rho_{y} \sigma_{\varepsilon_{y}} \sigma_{\varepsilon_{u}} \\
\rho_{u} \sigma_{\varepsilon_{y}} \sigma_{\varepsilon_{u}} & \sigma_{\varepsilon_{u}}^{2}
\end{array}\right)\right)
\end{gathered}
$$

y $\Phi(L)$ es un es un polinomio de rezagos bidimensional de orden "p".

\section{Dinámica transitoria autorregresiva (AR(2))}

A partir de la tradición establecida en los estudios sobre componentes no observables, el componente cíclico se modeló como un proceso autorregresivo $(\operatorname{AR}(2))$, ya que esto facilita el cumplimiento de la restricción de dejar fuera del círculo unitario a las raíces del polinomio autorregresivo durante la estimación de máxima verosimilitud (véanse, entre otros, Morley, Nelson y Zivot, 2003; Clark, 1987 y 1989; Watson, 1986) ${ }^{15}$. El modelo AR(2) se obtiene de la ecuación (5) eligiendo $\phi_{y}(L)=1-\phi_{1 y} L-\phi_{2 y} L^{2}, \phi_{u}(L)=1-\phi_{1 u} L-\phi_{1 u} L^{2}$. Se supone que las innovaciones $\left(\eta_{y t}, \eta_{u t}, \varepsilon_{y t}\right.$, y $\left.\varepsilon_{u t}\right)$ son variables aleatorias con distribución normal, con media igual a cero y una matriz de covarianza general, lo que permite establecer correlaciones entre cualesquiera de los componentes.

El modelo de componentes no observables se puede estimar mediante el uso de técnicas de modelos de estado-espacio para poder determinar la función de verosimilitud de la muestra. Si se supone que los términos de error tienen una distribución normal, los parámetros del modelo se pueden estimar mediante el método de máxima verosimilitud. Por ejemplo, la estimación de los parámetros en el sistema anterior puede obtenerse a partir de valores iniciales para el vector de estado y su matriz de covarianza. A partir de las estimaciones iniciales de los parámetros, el filtro de Kalman genera de manera recursiva las ecuaciones de predicción y,

15 La justificación teórica de la aplicación del ciclo AR(2) al desempleo está dada por Alogoskoufis y Manning (1988), quienes sostienen que la tasa de desempleo de todos los países se debería calcular mediante el modelo AR(2). también, los dos componentes no observables: $\tau_{i_{t}}, i=y, u$ y $c_{i}, i=y, u$. Una característica distintiva del modelo de estado-espacios es que la matriz de transición tiene dos raíces unitarias, que corresponden a las dos tendencias estocásticas. Por lo tanto, la matriz de covarianza de los valores iniciales del vector de estado es no acotada, lo que obliga a ser muy prudente en la inicialización. Para resolver este problema se recurrió al método de inicialización desarrollado por Koopman (1997) y refinado por Durbin y Koopman (2001) ${ }^{16}$.

Es probable que la identificación del modelo de componentes no observables no sea inmediata, pero la representación en su forma reducida ha permitido demostrar en varios artículos que es posible identificar un modelo de componente no observable con innovaciones correlacionadas, siempre que la complejidad de su dinámica lo permita (véanse, entre otros, Schleicher, 2003; Morley, Nelson y Zivot, 2003; Morley, 2007). Schleicher (2003) describe en términos generales algunos de los problemas técnicos que plantean la identificación y la estimación de un modelo multivariado y correlacionado de componentes no observables con innovaciones correlacionadas. Este autor demuestra que, en general, el requisito para identificar un modelo de componente no observable estructural caracterizado por tendencias y ciclos no comunes, en el que los componentes transitorios responden a un modelo de ciclos AR(p), se expresa como $p \geq 1+\frac{1}{n}$. Por lo tanto, en análisis multivariados los ciclos AR(2) implican una sobreidentificación del modelo. Cabe señalar que, aun cuando se identifique el modelo de componentes no observables con innovaciones no correlacionadas, si la identificación es débil podría causar problemas, dado que puede conducir a inferencias distorsionadas a través de las desviaciones estándar estimadas. Nelson y Startz (2007) demuestran que la varianza poblacional tiende a infinito si el modelo es no identificable, pero que la varianza de la muestra sigue siendo finita. Los autores recomiendan usar pruebas de razón de verosimilitud, en lugar del estadístico de Wald, para probar hipótesis cuando la identificación del modelo sea un problema potencial.

${ }^{16}$ En el modelo de componentes no observables, el vector de estado inicial difuso puede definirse de la siguiente manera: $\alpha_{1 \mid 0}=\left[\begin{array}{c}\delta_{k x 1} \\ 0_{2 l x 1}\end{array}\right]+v_{0}$, donde $\delta \sim N\left(0, k I_{2}\right), k \rightarrow \infty$. La matriz de covarianza del vector de estado inicial, $P_{1 \mid 0}$, puede dividirse en un componente no acotado, $k P_{\infty}$, correspondiente a las tendencias estocásticas, y un componente acotado, correspondiente al componente estacionario $P_{*}$. 
El modelo de caminata aleatoria-AR(2) implica los siguientes momentos:

$$
\begin{aligned}
& \operatorname{Var}\left(c_{y_{t}}\right)= \frac{\left(1-\phi_{2 y}\right) \sigma_{\varepsilon_{y}}^{2}}{\left(1+\phi_{2 y}\right)\left[\left(1-\phi_{2 y}\right)^{2}-\phi_{1 y}^{2}\right]^{\prime}} \\
& \operatorname{yar}\left(c_{u_{t}}\right)= \frac{\left(1-\phi_{2 u}\right) \sigma_{\varepsilon_{u}}^{2}}{\left(1+\phi_{2 u}\right)\left[\left(1-\phi_{2 u}\right)^{2}-\phi_{1 u}^{2}\right]^{\prime}} \\
& \frac{\operatorname{Cov}\left(c_{y_{t}}, c_{u_{t}}\right)=}{1-\phi_{1 y} \phi_{1 u}\left(1+\phi_{2 y} \phi_{2 u}\right)-\phi_{2 y}\left(\phi_{1 y}^{2}+2 \phi_{2 y}\right)-\phi_{2 y} \phi_{1 u}^{2}+\phi_{2 y}^{2} \phi_{2 u}^{2}}
\end{aligned}
$$

A continuación, se analiza el modelo en el contexto de la ley de Okun, quien indica que la brecha del producto y la brecha del desempleo están estrechamente relacionadas. Como la relación es esencialmente bidireccional, los investigadores han combinado las ecuaciones de regresión para estudiar la relación existente entre el producto causado por el desempleo (Freeman, 2001) y el desempleo causado por el producto (Sögner y Stiassny, 2000). Sin embargo, la interpretación de los resultados ha confundido en muchos casos a los autores y al mismo Okun, conduciéndolos a conclusiones espurias.

Como se ha indicado, la relación entre el producto real y la tasa de desempleo no es necesariamente lineal, lo que impone la necesidad de hacer dos regresiones para determinar las relaciones entre: desempleo causando al producto y el producto causando al desempleo, como se muestra a continuación:

$$
y_{t}-y *_{t}=\lambda\left(u_{t}-u *_{t}\right)+\vartheta_{t}
$$

o

$$
u_{t}-u *_{t}=\theta\left(y_{t}-y *_{t}\right)+\zeta_{t}
$$

donde $\left(y_{t}-y *_{t}\right)$ y $\left(u_{t}-u *_{t}\right)$ son los componentes transitorios del producto y la tasa de desempleo, respectivamente, y $\vartheta_{t}, \zeta_{t}$ representan los errores aleatorios. El mejor predictor lineal del desempleo a partir del producto se consigue calculando el desempleo en función del PIB (véase la ecuación 10); en cambio, para predecir el producto a partir del desempleo se debe realizar un análisis de regresión del PIB en función del desempleo (véase la ecuación 9).

El coeficiente de Okun se representa comúnmente como $\lambda$. La aplicación de métodos convencionales para su cálculo tiene dos consecuencias, como ya se ha dicho. En primer lugar, mediante MCO es sesgada e inconsistente $\mathrm{y}$, como $\lambda$ es negativo, $\hat{\lambda}$ tiende a dar un $\lambda$ sobreestimado. En segundo término, dada la correlación existente entre los dos componentes, es más adecuado estimar conjuntamente los dos componentes cíclicos. En el presente modelo, $\lambda$ y $\theta$ se calculan de la siguiente manera:

$$
\lambda=\frac{\operatorname{Cov}\left(c_{y_{t}}, c_{u_{t}}\right)}{\operatorname{Var}\left(c_{u_{t}}\right)}
$$

y

$$
\theta=\frac{\operatorname{Cov}\left(c_{y_{t}}, c_{u_{t}}\right)}{\operatorname{Var}\left(c_{y_{t}}\right)}
$$

\section{V}

\section{Resultados empíricos}

\section{Datos utilizados}

Las variables clave son el desempleo y la producción. Los datos sobre el PIB de México provienen del Instituto Nacional de Estadística y Geografía (INEGI) ${ }^{17}$, donde se calculan trimestralmente en pesos reales (año

\footnotetext{
17 La labor estadística del INEGI es comparable a la que realizan en los Estados Unidos de América la Oficina del Censo, la Oficina de Estadísticas Laborales y la Oficina de Análisis Económicos.
}

base $=2003$ ). Las series de desempleo se basan en la información recopilada en la Encuesta Nacional de Empleo Urbano (ENEU) y la Encuesta Nacional de Ocupación y Empleo (ENOE) que realiza el INEGI ${ }^{18}$. Las cifras corresponden a las áreas urbanas de México,

18 Los datos sobre desempleo que abarcan el período comprendido entre el primer trimestre de 1987 y el cuarto de 2004 provienen de la ENEU y fueron estandarizados de acuerdo con los criterios aplicados en la ENOE. 
que representan alrededor de una tercera parte de la población ${ }^{19}$. Todos los datos son trimestrales, han sido desestacionalizados y cubren el período comprendido entre el primer trimestre de 1987 y el cuarto trimestre de 2008.

En el gráfico 1 se ilustra la evolución de las variables utilizadas en el análisis. A fines de los años ochenta y mediados de la década de 2000, México registró altas tasas de desempleo junto con un aumento relativamente lento y escaso del nivel de producción. La crisis financiera que afectó al país en 1994 y la crisis mundial de 2008 provocaron un agudo incremento del desempleo y un drástico descenso del nivel de producción. La tasa promedio de desempleo del período 1987-2008 fue de alrededor de un $4,99 \%$, en tanto que los valores mínimo y máximo fueron de un 3,06\% y un $9,03 \%$, respectivamente. A comienzos de los años noventa, la tasa de desempleo mostró una leve tendencia ascendente

19 Cerca del $70 \%$ de la población de México vive en áreas urbanas. La situación demográfica y las condiciones imperantes en el mercado de trabajo de las áreas urbanas y rurales son muy diferentes, lo que debe tenerse en cuenta en la consideración de las conclusiones del presente estudio. y alcanzó su nivel máximo a fines de 1995. En 1996, el desempleo comenzó a descender rápidamente, hasta llegar a su nivel más bajo al término del año 2000, pero esta situación no se prolongó por mucho tiempo, porque ya en 2001 empezó a subir nuevamente. Esta tendencia ascendente de la tasa de desempleo podría atribuirse a la desaceleración de la economía de los Estados Unidos de América en 2001, que se acentuó después de los ataques terroristas del 11 de septiembre y afectó notablemente a la economía de México. El crecimiento del PIB real se redujo de un 6,6\% en 2000 a un $0,2 \%$ en 2001.

\section{Prueba de raíz unitaria}

Antes de estimar los componentes permanentes y transitorios de cada serie de tiempo empleando el modelo del componente no observable, se debe determinar si las series son o no estacionarias. En vista de que el período analizado abarca la crisis financiera que afectó a México en 1994, la recuperación registrada en la segunda mitad del gobierno de Zedillo, la recesión del año 2001 y el descenso de la tasa de crecimiento en el gobierno de Fox, se utiliza la prueba endógena de raíz unitaria de Lee y Strazicich (2003) basada en el multiplicador

GRÁFICO 1

México: PIB real y desempleo, primer trimestre de 1987 cuarto trimestre de 2008

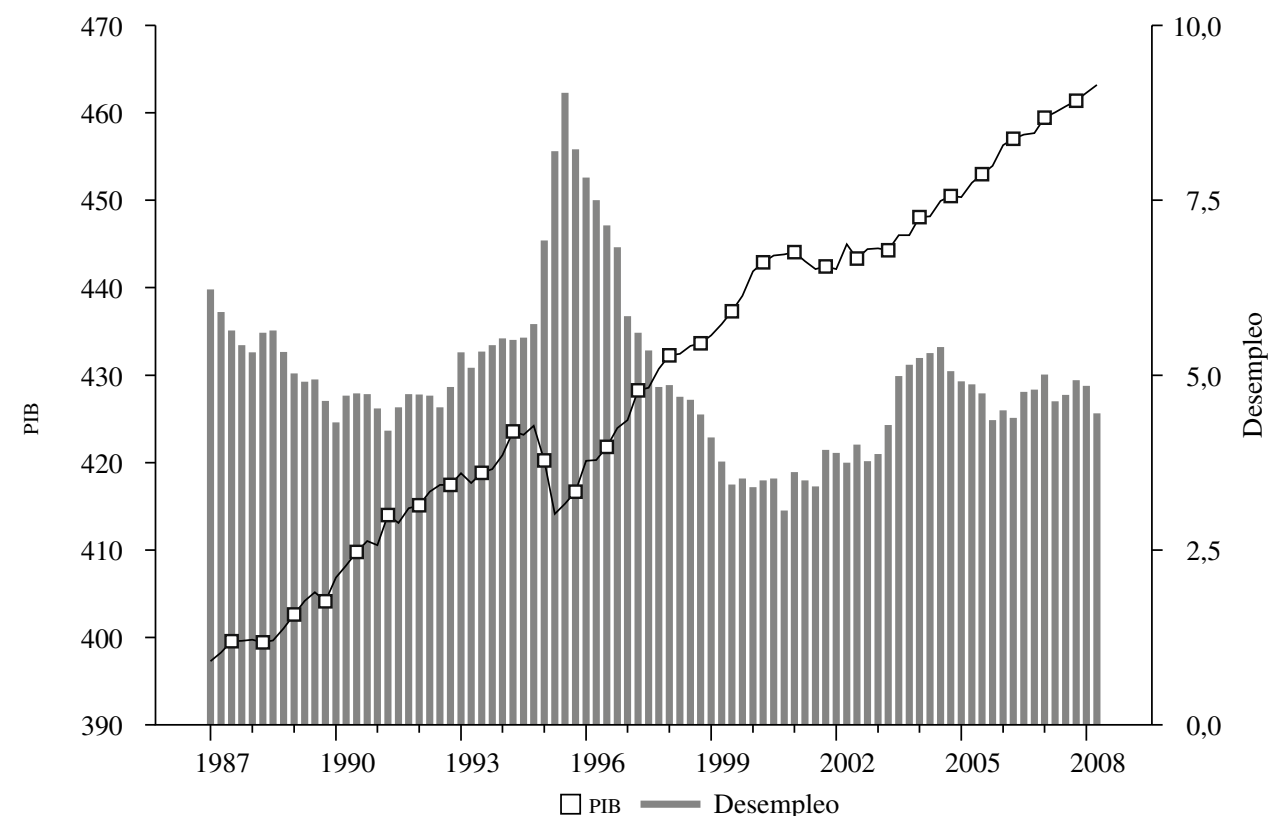

Fuente: Instituto Nacional de Estadística y Geografía (INEGI) de México.

PIB: producto interno bruto. 
mínimo de Lagrange con dos cambios estructurales. Los datos considerados son el logaritmo del PIB real multiplicado por $100\left(y_{t}\right)$ y la tasa de desempleo $\left(u_{t}\right)$. En el cuadro 1 se presentan los resultados de la prueba de raíz unitaria basada en datos de la serie en niveles. No se pudo rechazar la hipótesis nula, según la cual a cada serie correspondería una raíz unitaria, lo que significa que cada serie de tiempo presenta un proceso de raíz unitaria y, por lo tanto, sus niveles no son estacionarios. Esta es la condición deseable que permite aplicar el modelo de componentes no observables.

\section{Estimadores de máxima verosimilitud}

Usando el filtro de Kalman, se estiman los componentes no observables del producto y la tasa de desempleo mediante el método de máxima verosimilitud. En el cuadro 2 se presentan las estimaciones y sus correspondientes desviaciones estándar asintóticas, mientras que en los gráficos 2 y 3 se ilustran los componentes transitorios estimados de los logaritmos del PIB real y la tasa de desempleo, junto con los respectivos componentes permanentes no observables, cuyo cálculo se basó en el método de suavización de Kalman. En la aplicación de este método se utiliza toda la información de la muestra, lo que ofrece un mejor estimador que el filtro básico de Kalman, en el que solo se emplea la información disponible en el tiempo t. La deriva $\left(\mathrm{u}_{y}\right)$ del componente permanente del producto fue significativa, lo que no ocurrió con la deriva de la tasa de desempleo, por lo que no se reporta en los resultados. En la deriva del cuarto trimestre de 1994 se incluyó un cambio estructural en el logaritmo del PIB real.

A continuación se presentan algunas conclusiones del ejercicio que merecen ser destacadas.

En primer lugar, las innovaciones del componente permanente del producto tienen un impacto considerable y son más fuertes que choques similares en el componente permanente del desempleo. Al parecer, tanto las perturbaciones de la demanda como de la oferta influyen considerablemente en el comportamiento del producto. Por otra parte, debido a la rigidez del mercado laboral y al extenso mercado de trabajo informal, el desempleo abierto es menos sensible a las perturbaciones de origen externo.

En segundo término, las innovaciones de los componentes permanentes muestran una correlación significativa y negativa con las innovaciones de los componentes transitorios del PIB real y la tasa de desempleo. Asimismo, las estimaciones de los parámetros autorregresivos son relativamente bajas, lo que podría significar que gran parte de la persistencia de las dos series se concentra en el componente permanente. Como se observa en el cuadro 2, la mayoría de las variaciones del PIB real y de la tasa de desempleo provendrían de las perturbaciones permanentes.

En tercer lugar, y a diferencia de lo que ocurre con el producto, la volatilidad de los componentes

CUADRO 1

Prueba endógena de raíz unitaria con dos cambios estructurales, basada en el multiplicador de Lagrange

$\log$ (PIB). Modelo C: $\mathrm{K}=1, T_{B_{1}}=1994: 4, T_{B_{2}}=2000: 1, \mathrm{~N}=88, \lambda_{1} \cong 0,3, \lambda_{2} \cong 0,6$

Valores críticos $5 \%(-5,74) t_{\varnothing}=-3,5403$

\begin{tabular}{lllllccc}
\hline Parámetro & $\mu$ & $d_{1}$ & $d_{t_{1}}$ & $d_{2}$ & $d_{t_{2}}$ & $\phi$ \\
Estimador & 0,758 & $-4,123$ & $-0,596$ & 1,177 & 1,782 & $-0,2739$ \\
Estadístico t & $3,5403^{*}$ & $-3,2122^{*}$ & $-1,4205^{* *}$ & 0,930 & $3,305^{*}$ & $-3,5403$ \\
\hline
\end{tabular}

Desempleo. Modelo C: $\mathrm{K}=1, T_{B_{1}}=1995: 1, T_{B_{2}}=1999: 4, \mathrm{~N}=88, \lambda_{1} \cong 0,4, \lambda_{2} \cong 0,6$

Valores críticos $5 \%(-5,67) t_{\varnothing}=-2,865$

\begin{tabular}{lcccccc}
\hline Parámetro & $\mu$ & $d_{1}$ & $d_{t_{1}}$ & $d_{2}$ & $d_{t_{2}}$ & $\phi$ \\
Estimador & $-0,252$ & 1,555 & 0,023 & 0,249 & 0,393 & $-0,188$ \\
Estadístico t & $-2,302 *$ & $4,997 *$ & 0,184 & 0,783 & $4,120 *$ & $-2,865$ \\
\hline
\end{tabular}

Fuente: elaboración propia.

Nota: * y **: significancia del $5 \%$ y el $10 \%$, respectivamente.

Hipótesis nula: $y_{t}=\mu_{0}+d_{1} B_{1 t}+d_{t 1} D_{1 t}+\mathrm{d}_{2} B_{2 t}+d_{t 2} D_{2 t}+y_{t-1}+v_{1 t}$

Hipótesis alternativa: $y_{t}=\mu_{1}+\Upsilon t+d_{1} D_{1 t}+d_{t_{1}} D T_{1 t}+\mathrm{d}_{2} D_{2 t}+d_{t_{2}} D T_{2 t}+v_{2 t}$

Donde $D_{j t}=1$ para $t \geq T_{B j}+1, \mathrm{j}=1,2$ y 0 de lo contrario; $D T_{j t}=t-T_{B j}$ para $t \geq T_{B j}+1, j=1,2$ y 0 de lo contrario, $B_{j t}=1$ para $t=T_{B j}+1, j=1,2$, y 0 de lo contrario $T_{B j}$ se refiere al período en que se produce el cambio estructural. 
CUADRO 2

Estimadores de máxima verosimilitud del modelo con ciclos AR(2) ${ }^{a}$

\begin{tabular}{|c|c|c|c|c|c|}
\hline Parámetro & Estimación & Parámetro & Estimación & Parámetro & Estimación \\
\hline \multicolumn{2}{|c|}{ PIB real } & \multicolumn{2}{|c|}{ Tasa de desempleo } & \multicolumn{2}{|c|}{ Correlación cruzada } \\
\hline$\sigma_{\eta_{y}}$ & $\begin{array}{c}1,8487 \\
(0,3067)\end{array}$ & $\sigma_{\eta_{u}}$ & $\begin{array}{c}0,5141 \\
(0,1067)\end{array}$ & $\rho_{\eta_{y} \eta_{u}}$ & $\begin{array}{l}-0,7977 \\
(0,0670)\end{array}$ \\
\hline$\sigma_{\varepsilon_{y}}$ & $\begin{array}{c}0,8311 \\
(0,2676)\end{array}$ & $\sigma_{\varepsilon_{u}}$ & $\begin{array}{c}0,5105 \\
(0,1209)\end{array}$ & $\rho_{\eta_{y} \varepsilon_{u}}$ & $\begin{array}{c}0,7207 \\
(0,0816)\end{array}$ \\
\hline$\rho_{\eta_{y} \varepsilon_{y}}$ & $\begin{array}{l}-0,8151 \\
(0,1166)\end{array}$ & $\rho_{\eta_{u} \varepsilon_{u}}$ & $\begin{array}{l}-0,9929 \\
(0,0054)\end{array}$ & $\rho_{\eta_{u} \varepsilon_{y}}$ & $\begin{array}{c}0,9995 \\
(0,0054)\end{array}$ \\
\hline$\mu_{y_{1987-1994}}$ & $\begin{array}{c}0,7232 \\
(0,1212)\end{array}$ & $\phi_{1 u}$ & $\begin{array}{c}0,3267 \\
(0,1915)\end{array}$ & $\rho_{\varepsilon_{y} \varepsilon_{u}}$ & $\begin{array}{l}-0,9890 \\
(0,0338)\end{array}$ \\
\hline$\mu_{y_{1995-2010}}$ & $\begin{array}{c}0,7982 \\
(0,1903)\end{array}$ & $\phi_{2 u}$ & $\begin{array}{l}-0,0668 \\
(0,0967)\end{array}$ & & \\
\hline$\phi_{1 y}$ & $\begin{array}{c}0,4479 \\
(0,1673)\end{array}$ & & & & \\
\hline$\phi_{2 y}$ & $\begin{array}{l}-0,4000 \\
(0,1098)\end{array}$ & & & & \\
\hline
\end{tabular}

Logaritmo de la verosimilitud $=-158,2011$

Fuente: elaboración propia.

a $\sigma_{\eta_{i}} ; i=y, u$ : desviación estándar de las innovaciones permanentes.

$\sigma_{\varepsilon_{i}} ; i=y, u$ : desviación estándar de la innovaciones transitorias.

$\rho_{\eta_{i} \varepsilon_{i}} ; i=y, u$ : correlación entre innovaciones.

$\rho_{\eta_{v} \eta_{u}}$ : correlación entre el desempleo permanente y el PIB permanente.

$\rho_{\eta_{y} \varepsilon_{u}}$ : correlación entre el PIB permanente y el desempleo transitorio.

$\rho_{\eta_{u} \varepsilon_{v}}$ : correlación entre el desempleo permanente y el PIB transitorio.

$\rho_{\varepsilon_{y} \varepsilon_{u}}$ : correlación entre el PIB transitorio y el desempleo transitorio.

$\phi_{j i} ; i=y, u ; j=1,2$ : parámetros del modelo $\mathrm{AR}(2)$.

GRÁFICO 2

México: PIB real y componentes estimados, primer trimestre de 1987- cuarto trimestre de 2008

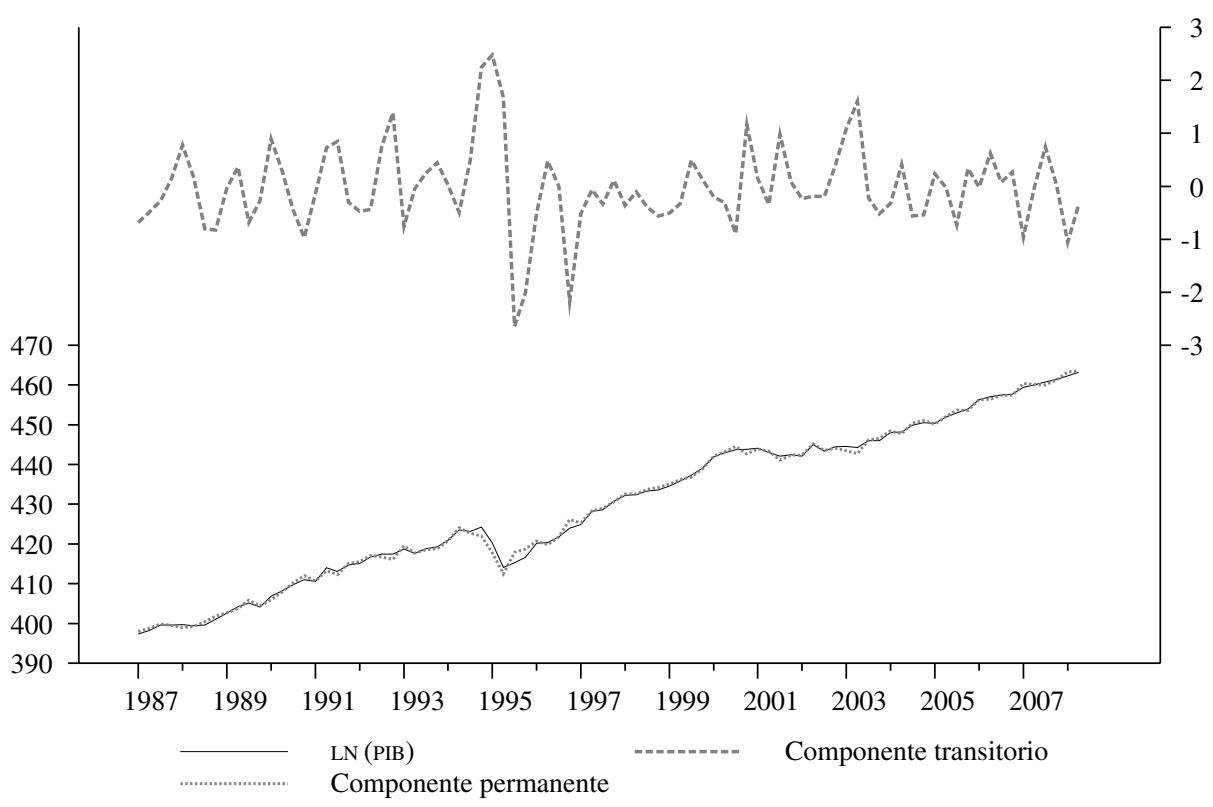

Fuente: elaboración propia.

LN (PIB): logaritmo natural del PIB. 
GRÁFICO 3

México: tasa de desempleo y componentes estimados, primer trimestre de 1987 - cuarto trimestre de 2008

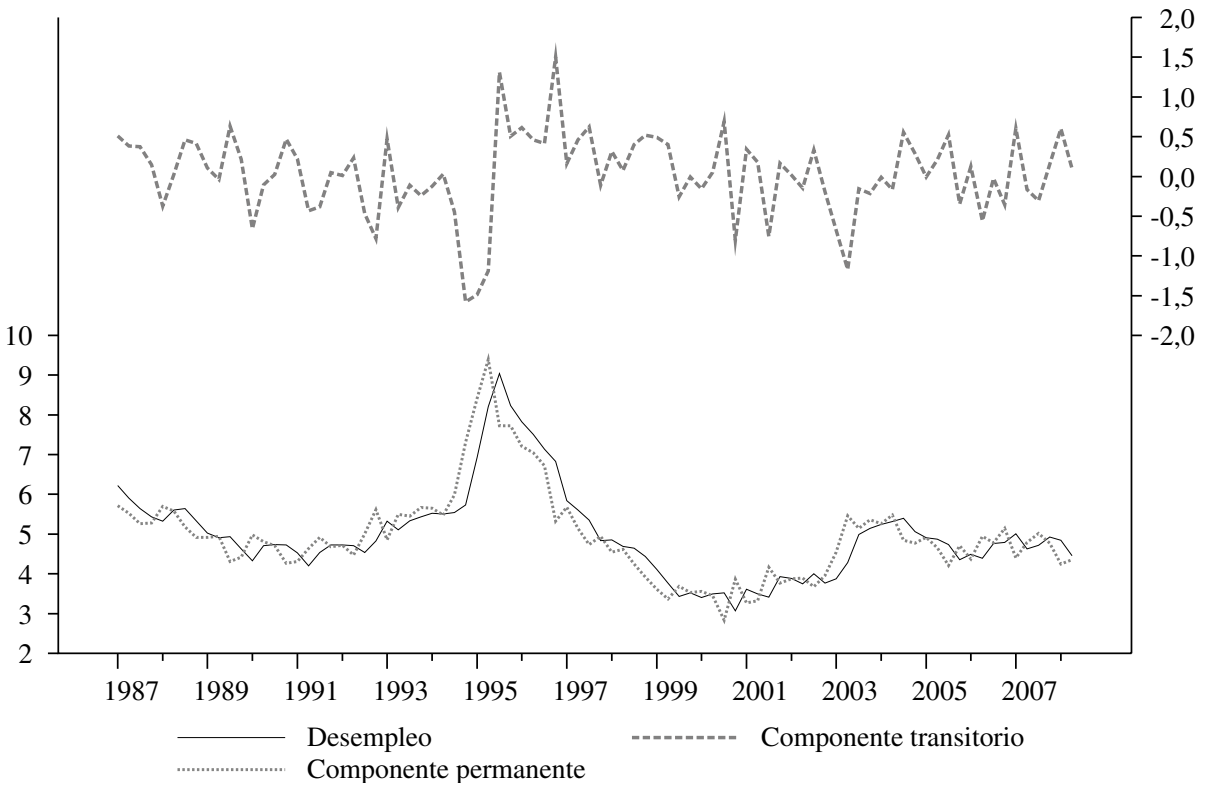

Fuente: elaboración propia.

transitorio y permanentes del desempleo es muy similar. Esto permitiría suponer que las perturbaciones externas que inciden en el mercado de trabajo afectan por igual a los dos tipos de componentes, aunque sus efectos son mucho menores en el caso del producto.

Un examen minucioso del gráfico 3 indicaría que al comienzo de la crisis financiera de 1994 la tasa de desempleo habría empezado a aumentar, y los cálculos efectuados en este estudio indican que su componente permanente creció en forma más acelerada en anticipación de su posible incremento en el futuro. La misma situación se observa a comienzos de 2001, cuando también se intensificó el alza del componente permanente de la tasa de desempleo, en anticipación del efecto negativo que podría tener para la economía de México la recesión en los Estados Unidos de América.

En cuarto lugar, de acuerdo con la ley de Okun, los componentes transitorios del producto y de la tasa de desempleo tienen una correlación negativa. Aunque los resultados del presente estudio demuestran que la mayor parte de las fluctuaciones del PIB real y de la tasa de desempleo obedecen a variaciones de sus componentes permanentes, es importante examinar la relación entre sus componentes transitorios.

La ecuación (9) es de gran ayuda para determinar el nivel esperado del PIB en las condiciones imperantes durante el período analizado, dado un cierto nivel de desempleo. Con tal objeto se realizó una estimación del coeficiente de Okun mediante la ecuación (11), cálculo que arrojó el siguiente resultado:

$$
\lambda=-1,657 \text { (desviación estándar: 0,842) }
$$

Esto significa que una disminución de un punto porcentual del empleo transitorio corresponde a un aumento de 1,6 punto porcentual del PIB real transitorio. Por lo tanto, la estimación del coeficiente de Okun $\lambda$ da un resultado muy inferior a los registrados anteriormente.

Hay varios factores que podrían explicar este coeficiente más bajo. En primer término, la estimación se basa en el supuesto de que el número de horas laborables y la productividad de la mano de obra evolucionan al mismo ritmo que el desempleo. Sin embargo, si la tasa de variación de cualquiera de estas variables fuera diferente, el coeficiente debería ser menor. Lamentablemente, no se cuenta con información confiable sobre ninguna de las variables que permita controlar estos cambios. En segundo lugar, como ya lo han consignado Heckman y Pagés (2000) y Gill, Montenegro y Dömeland (2001), entre otros, la Ley Federal del Trabajo de México y los sindicatos son los factores que explican la baja sensibilidad del empleo a las fluctuaciones del producto. Además, 
dado que el mercado de trabajo mexicano se caracteriza por la existencia de un extenso sector informal, las fluctuaciones del producto fomentan la movilidad entre el empleo formal e informal sin afectar al desempleo abierto $^{20}$. Todos estos elementos (las variaciones de las horas laborables, la productividad del trabajo, la estricta regulación laboral y la existencia de un amplio sector informal) operan en la misma dirección reduciendo el coeficiente de Okun.

En tercer lugar, como el empleo en el sector informal se caracteriza por una baja productividad, su contribución al PIB total es muy poco significativa. Por lo tanto, la disminución del desempleo no incidiría mayormente en el producto.

En cuarto lugar, la declaración de ingresos inferiores a los reales puede distorsionar las estimaciones del PIB. Loayza y Sugawara (2009) demostraron que la economía informal de México representa alrededor del $30 \%$ del PIB, en tanto que según las estimaciones de la Organización Internacional del Trabajo (OIT, 1999), Schneider (2002) y Vuletin (2008) esta representó entre un $30 \%$ y un $40 \%$ del PIB en los años noventa y absorbió porcentajes muy similares de la fuerza de trabajo.

En términos estrictamente keynesianos, la ecuación relevante debería ser la (10), en la que el desempleo es la variable dependiente y el producto es la variable independiente. En consecuencia, el coeficiente relevante es $\theta$ (véase la ecuación 12).

20 Cabe señalar que "sector informal" no es sinónimo de "empleo informal". El primero es el sector no regulado, mientras que el segundo es la modalidad de empleo que no da derecho a prestaciones (seguridad social, seguro de salud y vacaciones, entre otras). Por consiguiente, un trabajador puede tener un empleo informal en una empresa del sector formal.

$$
\theta=-0,5226 \text { (desviación estándar: -0,039) }
$$

Como era previsible, el valor de $\theta$ no está relacionado con el de $\lambda$. Mide el efecto de una fluctuación de un punto porcentual del incremento del producto en la variación del desempleo. Evidentemente, los factores considerados en el debate sobre la situación del mercado laboral de México son muy importantes en este contexto. Otro elemento que podría explicar la escasa correlación entre los componentes transitorios del producto y el desempleo es la migración de trabajadores. En la medida en que un gran número de trabajadores desempleados opta por emigrar a los Estados Unidos de América, en lugar de quedarse en México, un cambio en el producto afecta en menor grado a las tasas de desempleo registradas, lo que no se refleja en las estadísticas sobre la materia.

A continuación se analiza la correlación entre los componentes permanentes del producto y el desempleo. Para conocer la relación entre las innovaciones permanentes del producto y la tasa de desempleo se puede aplicar un raciocinio similar al empleado con respecto al coeficiente tradicional de Okun. Por lo tanto, si $\Upsilon$ es el coeficiente de Okun correspondiente a las fluctuaciones permanentes, $\gamma=\frac{\rho_{\eta_{y} \eta_{u}} \sigma_{\eta_{y}}}{\sigma_{\eta_{u}}}=-2,868$
(desviación estándar: 0,952).

El coeficiente calculado de los componentes permanentes del producto y el desempleo también es negativo, pero superior al de los componentes transitorios. Si en el cálculo del coeficiente se toman en consideración todos los ajustes a corto plazo, cabría esperar que fuera superior al coeficiente convencional de Okun (a corto plazo). $\mathrm{Al}$ parecer, la rigidez del desempleo provocada por los factores de rigidez del mercado de trabajo y la existencia del sector de empleo informal, que se manifiestan a corto plazo, sería menos determinante en períodos más largos.

\section{VI}

\section{Conclusiones}

El presente estudio tiene dos propósitos. El primero de ellos es estimar el coeficiente de Okun en la economía de México empleando un modelo bivariado para calcular conjuntamente los componentes permanentes y transitorios del producto y el desempleo, método que ofrece estimadores insesgados y eficientes. El segundo propósito es ofrecer una interpretación de los parámetros estimados. En el estudio se presentan dos visiones opuestas del mercado de trabajo mexicano y se explica que son importantes para comprender adecuadamente la magnitud del coeficiente de Okun.

La metodología utilizada permitió estimar dos coeficientes de Okun: un coeficiente a corto plazo de los componentes transitorios del producto y el desempleo y un coeficiente a largo plazo, derivado de la correlación de sus componentes permanentes. Los resultados obtenidos 
indican que el coeficiente de Okun es mucho más bajo que lo estimado anteriormente. Como se señaló en este artículo, esto era previsible, en parte debido a los factores de rigidez que presenta el mercado de trabajo de México y a la existencia de un extenso mercado de trabajo informal que reduce la influencia potencial del incremento del producto en las tasas de desempleo abierto. Otro factor que incide en este bajo coeficiente es el hecho de que los sindicatos limitan considerablemente la creación de empleos en períodos de expansión y evitan los recortes de empleos en los episodios de recesión. A largo plazo, también hay otros factores que intervienen en el mercado laboral mexicano, por lo que la correlación entre los componentes permanentes del producto y el desempleo es superior al coeficiente a corto plazo. Se trata concretamente de los cambios en la utilización de la capacidad productiva, el progreso técnico, el carácter cambiante de los contratos de trabajo, la migración internacional y los cambios demográficos, factores todos que contribuirían a elevar el coeficiente. En todo caso, estos temas podrían seguir investigándose en el futuro.
En el presente estudio se ha presentado una estimación del coeficiente de Okun en un período determinado. Sin embargo, debido al carácter cambiante del mercado de trabajo de México y, en particular, al aumento de los contratos a corto plazo y las subcontrataciones externas - fenómenos documentados con un número cada vez mayor de evidencias- es muy probable que el coeficiente no se haya mantenido constante. A juicio de los autores de este artículo, es posible que la relación dinámica entre los componentes permanentes y cíclicos haya ido cambiando, lo que reviste especial importancia en vista de la nueva estrategia económica adoptada por México a mediados de los años ochenta, estrategia que no solo ha supuesto una liberalización comercial y de los precios, sino también cambios en las instituciones del mercado laboral, entre otras cosas. Además, queda por determinar si el coeficiente de Okun es simétrico, es decir, si cabe o no esperar que el incremento del producto influya de igual manera en el desempleo tanto en los episodios de expansión como de recesión.

\section{Bibliografía}

Alcaraz, C. (2009), "Informal and formal labour fexibility in Mexico", Desarrollo y Sociedad, $\mathrm{N}^{\circ}$ 63, Bogotá, Centro de Estudios sobre Desarrollo Económico (CEDE).

Alcaraz, C., D. Chiquiar y M. Ramos-Francia (2008), "Diferenciales salariales intersectoriales y el cambio en la composición del empleo urbano de la economía mexicana en 2001-2004", Documentos de Investigación, $\mathrm{N}^{\circ}$ 2008-06, México, D.F., Banco de México.

Alogoskoufis, G. y A. Manning (1988), "Wage setting and unemployment persistence in Europe, Japan and the USA", European Economic Review, vol. 32, $\mathrm{N}^{\circ} 3$, Amsterdam, Elsevier.

Balakrishnan, R., M. Das y P. Kannan (2010), "La dinámica del desempleo durante las recesiones y las recuperaciones: La Ley de Okun como punto de partida", Perspectivas de la economía mundial, Washington, D.C., Fondo Monetario Internacional.

Barreto, H. y F. Howland (1993), "There are two Okun's law relationships between output and unemployment", Crawfordsville, Wabash College.

Blanchard, O. y D. Quah (1989), "The dynamic effects of aggregate demand and supply disturbances", American Economic Review, vol. 79, $\mathrm{N}^{\circ}$ 4, Nashville, Tennessee, American Economic Association.

Calderón, A. (2000), "Job stability and labor mobility in Urban Mexico: a study based on duration models and transition analysis", Research Department Publications, $\mathrm{N}^{\circ} 3117$, Washington, D.C., Banco Interamericano de Desarrollo.

Cazes, S., S. Verick y F. Al Hussami (2011), "Diverging trends in unemployment in the United States and Europe: evidence from Okun's law and the global financial crisis", Employment Working Paper, $\mathrm{N}^{\circ} 106$, Ginebra, Oficina Internacional del Trabajo.

Chavarín, R. (2001), "El costo del desempleo medido en producto. Una revisión empírica de la ley de Okun para México", $E l$ Trimestre Económico, vol. 68, N 270, México, D.F., Fondo de Cultura Económica.
Clark, P. (1989), "Trend reversion in real output and unemployment", Journal of Econometrics", vol. 40, $\mathrm{N}^{\circ} 1$, Amsterdam, Elsevier. (1987), "The cyclical component of U.S. economic activity", Quarterly Journal of Economics, vol. 102, N ${ }^{\circ}$ 4, Cambridge, Massachusetts, The MIT Press, noviembre.

Crespo, J. (2003), "Okun's law revisited", Oxford Bulletin of Economics and Statistics, vol. 65, $\mathrm{N}^{\circ} 4$, Wiley.

De la Garza, E. (2005), "La flexibilidad del trabajo en México" [en línea] http://docencia.izt.uam.mx/egt/publicaciones/articulos/ flexibilidad.pdf.

Durbin, J. y S. Koopman (2001), Time Series Analysis by State Space Methods, Nueva York, Oxford University Press.

Evans, G.W. (1989), "Output and unemployment dynamics in the United States: 1950-1985”, Journal of Applied Econometrics, vol. 4, $\mathrm{N}^{\circ} 3$, John Wiley \& Sons, Ltd.

Freeman, D.G. (2001), "Panel test of Okun's law for ten industrial countries", Economic Inquiry, vol. 39, ํㅜ 4, Wiley.

Gill, I., C. Montenegro y D. Dömeland (eds.) (2001), "Crafting Labor Policy: Techniques and Lessons Learned from Latin America", Washington, D.C., Banco Mundial.

González Anaya, J. (2002), "Labor Market Flexibility in Thirteen Latin American Countries and the United States: Revisiting and Expanding Okun Coefficients", SCID Working Paper, $\mathrm{N}^{\circ} 136$, Universidad de Stanford, junio.

Heckman, J. y C. Pagés (2000), "The Cost of Job Security Regulation: Evidence From Latin American Labor Markets", NBER Working Paper, $\mathrm{N}^{\circ} 7773$, Cambridge, Massachusetts, National Bureau of Economic Research.

Hwa, K., E. Zivot y D. Creal (2006), "The relationship between the Beveridge-Nelson decomposition and unobserved components models with correlated shocks", Working Papers, $\mathrm{N}^{\circ}$ UWEC2006-16-FC, Seattle, Universidad de Washington.

Jardin, M. y S. Gaétan (2011), "How Okun's law is non-linear in Europe: a semi-parametric approach", Rennes, University of Rennes 1 - CREM, mayo. 
Knotek, E.S. (2007), "How useful is Okuns's law?", Economic Review, Kansas, Banco de la Reserva Federal de Kansas.

Koopman, S. (1997), "Exact initial Kalman filtering and smoothing for non-stationary time series models", Journal of the American Statistical Association, vol. 92, $\mathrm{N}^{\circ}$ 440, Taylor \& Francis.

Lee, J. (2000), "The robustness of Okun's law: evidence from OECD countries", Journal of Macroeconomics, vol. 22, $\mathrm{N}^{\circ} 2$, Amsterdam, Elsevier.

Lee, J. y M. Strazicich (2003), "Minimum Lagrange multiplier unit root test with two structural breaks", The Review of Economics and Statistics, vol. 85, $\mathrm{N}^{\circ}$ 4, Cambridge, Massachusetts, The MIT Press.

Loayza, N. y N. Sugawara (2009), "El sector informal en México: Hechos y explicaciones fundamentales", El Trimestre Económico, vol. 76, $\mathrm{N}^{\circ}$ 4, México, D.F., Fondo de Cultura Económica.

Loria, E. y M. Ramos (2007), "La ley de Okun: Una relectura para México, 1970-2004”, Estudios Económicos, vol. 22, № 1, México, D.F., El Colegio de México.

Marshall, A. (2004), "Labour market policies and regulations in Argentina, Brazil and Mexico: programmes and impacts", Buenos Aires, Consejo Nacional de Investigaciones Científicas y Técnicas/Instituto de Desarrollo Económico y Social.

Morley, J.C. (2007), "The slow adjustment of aggregate consumption to permanent income", Journal of Money, Credit, and Banking, vol. 39, $\mathrm{N}^{\circ} 2-3$, Blackwell Publishing.

Morley, J.C., C.R. Nelson y E. Zivot (2003), "Why are the BeveridgeNelson and unobserved components decompositions of GDP so different?", Review of Economics and Statistics, vol. 85, Nㅜㄹ, Cambridge, Massachusetts, The MIT Press.

Nelson, C.R. y C.I. Plosser (1982), "Trends and random walks in macroeconomic time series: some evidence and implications", Journal of Monetary Economics, vol. 10, Nㅜ 2, Amsterdam, Elsevier.
Nelson, C.R. y R. Startz (2007), "The zero-information-limit condition and spurious inference in weakly identified models", Journal of Econometrics, vol. 138, $\mathrm{N}^{\circ} 1$, Amsterdam, Elsevier.

OIT (Organización Internacional del Trabajo) (1999), Panorama laboral 1999, Lima.

Okun, A.M. (1962), "Potential GNP: its measurement and significance", Proceedings of the Business and Economic Statistics Section, Washington, D.C., American Statistical Association.

Prachowny, M. (1993), "Okun's law: theoretical foundations and revised estimates", Review of Economics and Statistics, vol. 75, $\mathrm{N}^{\circ}$ 2, Cambridge, Massachusetts, The MIT Press, mayo.

Schleicher, Ch. (2003), "Structural time-series models with common trends and common cycles", University of British Columbia.

Schnabel, Gert (2002), "Output trends and Okun's law", BIS Working Papers, $\mathrm{N}^{\circ} 111$, Basilea, Banco de Pagos Internacionales, abril.

Schneider, F. (2002), "Size and measurement of the informal economy in 110 countries around the world", Washington, D.C., Banco Mundial.

Sinclair, T. (2009), "The relationships between permanent and transitory movements in U.S. output and the unemployment rate", Journal of Money Credit and Banking, vol. 41, $\mathrm{N}^{\circ} 2-3$, Blackwell Publishing, marzo-abril.

Sögner, L. y A. Stiassny (2000), “A cross-country study on Okun's law", Working Paper Series, N ${ }^{\circ} 13$, Viena, Vienna University of Economics and Business.

Vuletin, G. (2008), "Measuring the informal economy in Latin America and the Caribbean", IMF Working Paper, $\mathrm{N}^{\circ} 08 / 102$, Washington, D.C., Fondo Monetario Internacional.

Watson, M.W. (1986), "Univariate detrending methods with stochastic trends", Journal of Monetary Economics, vol. 18, $\mathrm{N}^{\circ} 1$, Amsterdam, Elsevier.

Weber, C. (1995), "Cyclical output, cyclical unemployment and Okun's coefficient: a new approach", Journal of Applied Econometrics, vol. 10, $\mathrm{N}^{\circ}$ 4, John Wiley \& Sons, Ltd. 